\title{
Adaptive Color Texture Image Segmentation Using $\alpha$-Cut Implemented Interval Type-2 Fuzzy C-Means
}

\author{
${ }^{1} \mathrm{P}$. Murugeswari and ${ }^{2} \mathrm{D}$. Manimegalai \\ ${ }^{1}$ Department of IT, Sri Vidya College of Engineering and Technology, \\ Sivakasi Main Road, Virudhunagar, Tamil Nadu, India \\ ${ }^{2}$ Department of IT, National Engineering College, K.R. Nagar, \\ Kovilpatti, Tamil Nadu, India
}

\begin{abstract}
Texture segmentation is the process of partitioning an image into regions with different textures containing similar group of pixels. The color and texture information collectively has strong link with the human perception. So many applications need to combine color and texture features analyse the image content accurately. This study presents an unsupervised color texture image segmentation method which is based on the feature extraction and fuzzy clustering. The proposed method includes color texture segmentation using Haralick features extracted from Integrated Color and Intensity Co-occurrence Matrix (ICICM). Then, $\alpha$-cut implemented interval type-2 fuzzy c-mean clustering algorithm is utilized to cluster the obtained feature vectors into several classes corresponding to different regions of the textured image. Experimental result shows that the proposed hybrid approach could obtain better cluster quality and segmentation results compared to state of art image segmentation algorithms.
\end{abstract}

Key words: Type- 2 fuzzy, color texture segmentation, IT2 fuzzy, ICICM, CCM, India

\section{INTRODUCTION}

In computer vision and pattern recognition color texture image segmentation is one of the most important research areas since, it is a necessary step for high level image analysis such as object reorganization, scene understanding, scene detection and shot detection. Over the past three decades, many algorithms have been proposed by researchers: extracting the texture features in each color band and segment and extracting color and texture features on separate band and then combining them in the segmentation process. Based on review of literature it is understood that none of the algorithm segments all types of textures because algorithms are designed for a particular application. Therefore, still more algorithms are needed in this field to improve the accuracy, computational cost, etc. Ilea and Whelan (2011) have investigated the field of color image segmentation for over the past three decades and they have stated that nearly 1000 studies are published during 1984-2008. In early 1984-1992 approaches of segmentation, all algorithms are purely application driven. Based on the approach used in the extraction and integration of the color and texture features, three major trends are identified in color texture analysis. They are: implicit color texture feature integration, approaches that extract color and texture in successionl, approaches that extract the color and texture features on separate channels and then combine them in the segmentation process.

Zadeh first introduced the Type-1 Fuzzy Set (T1FS) theory in 1965 and has been successfully applied in many areas including image processing, modeling and control, data mining time-series prediction, etc. This review of literature focused color texture image segmentation based on fuzzy. Fuzzy is utilized to segment the image based on fuzzy clustering, fuzzy rule-based (fuzzy IF-THEN) technique, fuzzy geometry, fuzzy thresholding and fuzzy entropy. In these methods, clustering is a well known method which is a mathematical tool that attempts to discover structures or certain pattrens in data set where the objects inside each cluster show certain degree of similarity. Most widely used clustering method is the Fuzzy C-Means (FCM) technique which is applied in many image processing area such as medical image analysis and remote sensing. FCM was developed by Dunn in 1973 and improved by Bezdek in 1981 which has

Corresponding Author: P. Murugeswari, Department of IT, Sri Vidya College of Engineering and Technology, Sivakasi Main Road, Virudhunagar, Tamil Nadu, India 
been further developed as Modified FCM (Du and Lee, 2009), Suppressed FCM (Fan et al., 2003), Modified Suppressed FCM and Alternative FCM proposed by Yang and Wu (2003), Possibilistic FCM (Pal et al., 2005), Generalized FCM (Zhu et al., 2009), FCM with spatial information (Chuang et al., 2006) and FCM combined with ant colony algorithm (ACFHA) (Shokouhifar and Farokhi 2010). The advantages of FCM Method is straightforward implementation, fairly robust behavior and applicability to multichannel data and ability of uncertainty data modeling. However, the disadvantages of FCM is none of the data points have membership value 1 , noise and outliers may have difficulties in obtaining appropriate clustering results and it does not incorporate information about spatial context. Yang et al. (2008) proposed $\alpha$-cut implemented fuzzy clustering algorithm referred to as $\mathrm{FCM} \alpha$ which allows the data points being able to completely belong to one cluster. FCM $\alpha$ algorithm can form a cluster core for each cluster where data points inside a cluster core will have a value 1 . So it can resolve the drawbacks of FCM.

The concept of type-2 fuzzy set was introduced by Zadeh (1975) and it was further developed by Karnik et al. (2002). Type-2 fuzzy is the extension of type-1 fuzzy with an additional dimension. Type- 2 Fuzzy Set (T2FS) is bounded with above and below two T1FS which are called Upper Membership Function (UMF) and Lower Membership Function (LMF), respectively. The area between UMF and LMF is the Footprint of Uncertainty (FOU). So, T2 FS provides more design degrees of freedom for uncertainty. Now type-2 fuzzy sets prove to model various uncertainties but it increases the computational complexity because of its additional dimension of secondary grades for each primary membership. Example applications are type-2 fuzzy clustering (Tlig et al., 2010) Gaussian noise filter (Wang et al., 2005), classification of coded video streams (Liang and Mendel, 2001), medical applications (Innocent and John, 2002) and color image segmentation (Maity and Sil, 2009). Because of the computational complexity of using a general $\mathrm{T} 2 \mathrm{FS}$, many applications use an Interval Type-2 Fuzzy Set (IT2FS).

Background: Fuzzy C-Means (FCM) is a method of clustering which allows one piece of data to belong to two or more clusters and it is frequently used in pattern recognition. In unsupervised learning, FCM clustering algorithms are best known and most powerful methods used in cluster analysis. The FCM algorithm attempts to partition a finite collection of elements $X=\left\{x_{1}, x_{2}, \ldots, x_{n}\right\}$ into a collection of c fuzzy clusters with respect to some given criterion. When fuzzy clustering is attempted, fuzzy membership design includes various uncertainties such as distance measure, fuzzifier and prototype. This section reviews the models such as FCM and Type reduction using $\alpha$-plane representation which are proposed in Yang et al. (2008) and Liu (2008), respectively.

Fuzzy c-Means clustering algorithm using $\alpha$-cut technique (FCM $\alpha)$ : Set the iteration counter $1=0$ and choose initial cluster centers $v^{(0)}, i=1, \ldots, c$. Give a value of $\alpha$ with $0.5 \leq \alpha \leq 1$ and a termination value $\varepsilon>0$ :

$$
\begin{gathered}
\mu_{\mathrm{ij}}^{(1)}=\left(\sum_{\mathrm{k}=1}^{\mathrm{c}} \frac{\left\|\mathrm{x}_{\mathrm{j}}-v_{\mathrm{i}}\right\|^{2 /(\mathrm{m}-1)}}{\left\|\mathrm{x}_{\mathrm{j}}-v_{\mathrm{k}}\right\|^{2 /(\mathrm{m}-1)}}\right)^{-1}>\alpha \\
v_{\mathrm{i}}^{(1)}=\frac{\sum_{\mathrm{j}=1}^{\mathrm{n}} \mu_{\mathrm{ij}}^{\mathrm{m}} \mathrm{x}_{\mathrm{j}}}{\sum_{\mathrm{j}=1}^{\mathrm{n}} \mu_{\mathrm{ij}}^{\mathrm{m}}}, \mathrm{i}=1, \ldots, \mathrm{c}
\end{gathered}
$$

Step 1: Compute $\mu_{\mathrm{ij}}^{(1)}$ with Eq. 1 .

Step 2: Update $\mu_{i j}^{(1)}$ for $i=1, \ldots, c, j=1, \ldots, n$ by the following way: if $\max _{1 \leq k \leq c} \mu_{i j}{ }^{(1)} \leq \alpha$ then $\mu_{i j}{ }^{(1)}=\mu_{i j}{ }^{(1)}$ for all $i=1, \ldots, c$. If $\mu_{\mathrm{ij}}^{(1)}=\max _{1 \leq \mathrm{k} s \mathrm{c}} \mu_{\mathrm{ij}}^{(1)}>\alpha$, then $\mu_{\mathrm{ij}}^{(1)}=1$ and $\mu_{\mathrm{ij}}^{(1)}=0$ for all $\mathrm{i}^{\prime} \neq \mathrm{i}$

Step 3: Calculate $\mathrm{v}_{i}^{(1+1)}$ with Eq. 2

Step 4: If $\max _{i}\left\|v_{i}^{(1+1)}-v_{i}\right\|<\omega$ then stop; else $1=1+1$ and goto Step 1.

$\boldsymbol{\alpha}$-cut implemented type-2 fuzzy: In Hamrawi et al. (2010) the $\alpha$-cut decomposition theory is one of the fundamental concepts in the field of FSs. This $\alpha$-cut has the capable to decompose FSs into a collection of crisp sets. This decomposition along with the extension principle forms a methodology for extending mathematical concepts directly from crisp sets to FSs.

This $\alpha$-cut decomposition theory can be summarized in three main concepts: $\alpha$-plane extension principle, $\alpha$-cut decomposition theorem for T2FSs and $\alpha$-cut extension principle of T2FSs. Figure 1 shows the example of $\alpha$-cut implemented IT2FS.

Centriod type- 2 reduction using representation: One of the most important concepts in fuzzy set theory and applications is the $\alpha$-cut decomposition theorem developed by Zadeh (1975) under the name resolution identity. These cuts are crisp sets associated with certain 


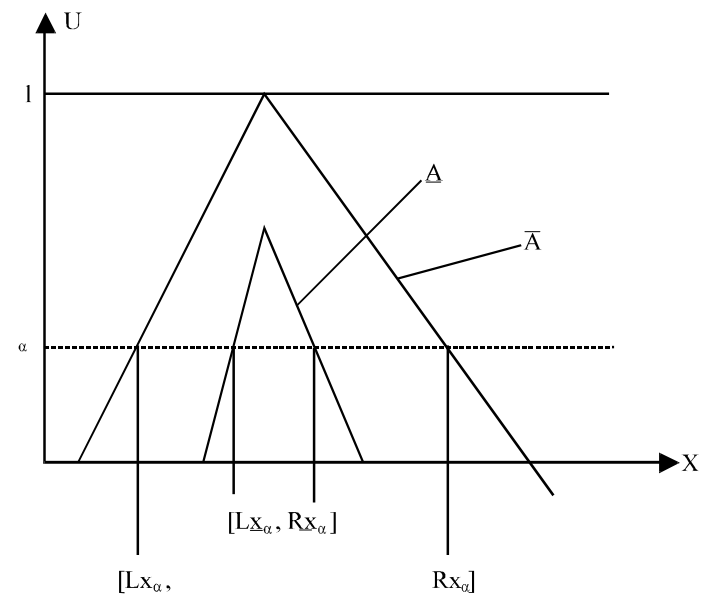

Fig. 1: IT2 FS with cut

$\alpha$ levels that represent distinct grades of membership. The idea behind the $\alpha$-cut representation is to define a useful special fuzzy set that is associated with each $\alpha$-cut. The Eq. 3 shows the $\alpha$-cut of type-1 fuzzy set:

$$
\mathrm{A}_{\alpha}=\{\mathrm{A} \in \mathrm{X} \mid \mathrm{A}(\mathrm{x}) \geq \alpha, \alpha \in[0,1]\}
$$

The two-dimensional $\alpha$-plane, denoted $\widetilde{\mathrm{A}}_{\alpha}$ the union of all primary membership show secondary grades are greater than or equal to the special value $\alpha$, i.e.

$$
\tilde{\mathrm{A}}_{\alpha}=\mathrm{U}_{\mathrm{zeE}}(\mathrm{x}, \mathrm{u}) \mid \mu_{\tilde{\mathrm{A}}}(\mathrm{x}, \mathrm{u}) \geq \alpha=\mathrm{U}_{\mathrm{zex}}(\mu \tilde{\mathrm{A}}(\mathrm{x}))_{\alpha}
$$

where, $\left(\mu_{\tilde{A}}(x)\right)_{\alpha}$ is the $\alpha$-cut of $\mu_{\tilde{A}}(x)$ vertical slice. The centroid of an interval type-2 fuzzy set is represented in Eq. 5:

$$
\begin{aligned}
v_{\mathrm{c}}(\alpha) & =\operatorname{Centroid}(\tilde{\mathrm{A}}(\alpha) \\
& =\sum_{\mathrm{u}_{1} \in \sigma J \mathrm{~J}_{1}}, \ldots \sum_{\mathrm{u}_{\mathrm{N}} \in \mathrm{JJJ}_{\mathrm{X}}} \alpha / \frac{\sum_{\mathrm{i}=1}^{\mathrm{N}} \mathrm{x}_{\mathrm{i}} \mathrm{u}_{\mathrm{i}}}{\sum_{\mathrm{i}=1}^{\mathrm{N}} \mathrm{u}_{\mathrm{i}}} \\
& =\alpha /\left[{ }^{\alpha} v_{1},{ }^{\alpha} v_{\mathrm{r}}\right]
\end{aligned}
$$

\section{MATERIALS AND METHODS}

The proposed methodology segments the color texture image into different regions using Haralick features extracted from ICICM. The images obtained from the MINERVA scene analysis benchmark database is in RGB Color Model. In order to measure properly, image colors are represented in a modified HSV color space. Then, the ICICM is calculated. From ICICM, the texture features are extracted. Finally, images are segmented using IT $2 \mathrm{FCM} \alpha$ clustering algorithm.
Extraction of color feature: Vadivel et al. (2007) proposed an integrated approach for capturing spatial variation of both color and intensity levels in the neighborhood of each pixel using HSV color space. In their study, they have estimated the amount of color and intensity variation between each pixel and its neighbour using weight function. Suitable weighted constraints are satisfied while choosing the weight function for effectively relating visual perception of color and the HSV color space properties. Therefore, ICICM is generalization of grayscale co-occurrence matrix and Color Co-occurrence Matrix (CCM) technique. The advantage of ICICM is the color and intensity variations are represented in a single composite feature. Some of the useful properties of the HSV color space and their relationship to human color perception are utilized for extracting this feature.

The generation of ICICM algorithm is summarized: ICICM is two-dimensional matrix which consists of four sub matrix and it can be represented as follows:

$$
\left(\begin{array}{ll}
\mathrm{ICICM}_{\mathrm{CC}} & \mathrm{ICICM}_{\mathrm{CI}} \\
\mathrm{ICICM}_{\mathrm{IC}} & \mathrm{ICICM}_{\mathrm{II}}
\end{array}\right)
$$

Where:

$\mathrm{ICICM}_{\mathrm{CC}}=$ Color perception of pixel $\mathrm{p}$ and color perception of its neighbour $\mathrm{Np}$

$\mathrm{ICICM}_{\mathrm{CI}}=$ Color perception of pixel $\mathrm{p}$ and gray level perception of its neighbour $\mathrm{Np}$

$\mathrm{ICICM}_{\mathrm{IC}}=$ Gray level perception of pixel $\mathrm{p}$ and color perception of its neighbour $\mathrm{Np}$

$\mathrm{ICICM}_{\mathrm{II}}=$ Gray level perception of pixel p and gray level perception of its neighbour $\mathrm{Np}$

The dimension of the matrix ICICM is determined by the number of quantization levels of $\mathrm{HL}$ and GL; it can be computed as follows:

$$
\begin{aligned}
& \mathrm{HL}=\left\lfloor\frac{2 \pi}{\mathrm{Q}_{\mathrm{H}}}\right\rfloor+1 \\
& \mathrm{GL}=\left\lfloor\frac{255}{\mathrm{Q}_{1}}\right\rfloor+1
\end{aligned}
$$

where, QH and QI are quantization factors for Hue and Intensity. Each component of ICICM has been calculated as follows:

$$
\operatorname{ICICM}_{\mathrm{CC}}(\mathrm{i}, \mathrm{j})_{\mathrm{i}=0 . \mathrm{HL}-1 ; \mathrm{j}=0 . \mathrm{HL}-1}=\left(\left(\mathrm{hI}_{\mathrm{p}}, \mathrm{hI}_{\mathrm{Np}}\right)=(\mathrm{i}, \mathrm{j})\right)
$$




$$
\begin{aligned}
& \operatorname{ICICM}_{\mathrm{CI}}(\mathrm{i}, \mathrm{j})_{\mathrm{i}=0 . \mathrm{HL}-1 ; \mathrm{j}=0 . \mathrm{GL}-1}=\left(\left(\mathrm{hI}_{\mathrm{p}}, \mathrm{gI}_{\mathrm{Np}}\right)=(\mathrm{i}, \mathrm{j})\right) \\
& \operatorname{ICICM}_{\mathrm{IC}}(\mathrm{i}, \mathrm{j})_{\mathrm{i}=0 . \mathrm{GL}-1 ; \mathrm{j}=0 . \mathrm{HL}-1}=\left(\left(\mathrm{gI}_{\mathrm{p}}, \mathrm{hI}_{\mathrm{Np}}\right)=(\mathrm{i}, \mathrm{j})\right) \\
& \operatorname{ICICM}_{\mathrm{II}}(\mathrm{i}, \mathrm{j})_{\mathrm{i}=0 . \mathrm{GL}-1 ; \mathrm{j}=0 . \mathrm{GL}-1}=\left(\left(\mathrm{gI}_{\mathrm{p}}, \mathrm{gI}_{\mathrm{Np}}\right)=(\mathrm{i}, \mathrm{j})\right)
\end{aligned}
$$

Update the ICICM using weight function:

$$
\mathrm{W}_{\mathrm{col}}(\mathrm{S}, \mathrm{I})= \begin{cases}\mathrm{S}^{\left.\mathrm{ti}_{1} \times(255 \mid)^{2}\right)^{2}}, & \text { for } \mathrm{I} \neq 0 \\ 0, & \text { for } \mathrm{I} \neq 0\end{cases}
$$

where, $r_{1}$ and $r_{2}$ are constants which is dependent on the particular applications. The intensity weight of a pixel is computed as a complement of the color weight as given bellow:

$$
\mathrm{W}_{\text {int }}(\mathrm{S}, \mathrm{I})=1-\mathrm{W}_{\text {coI }}(\mathrm{S}, \mathrm{I})
$$

Extraction of texture feature: Haralick features are considered for extracting the properties from texture images. Haralick introduced 14 texture features extracted from CCM (Haralick et al., 1973). These features are statistical measures on the CCM of an image which allow reduction of information quantity of each matrix. Palm used 8 of these 14 Harlaick features namely homogeneity, contrast, correlation, variance, inverse difference moment, entropy, correction 1 and 2. Maheswari et al. (2011) used only 5 of these 14 Haralick features contrast, correlation, variance, inverse difference moment and angular second moment in order to reduce the size of the feature space. Generally, for each image coded in a color space it is needed to calculate $3 \mathrm{CCM}$ matrices and so $\mathrm{N}_{\mathrm{f}}=3 \times 14$ Haralick features are extracted from these matrices. The total number $\left(\mathrm{N}_{\mathrm{f}}\right)$ of candidate color texture features are very high. Since, it is needed to reduce the feature space, therefore, in the proposed method ICICM is calculated and $1 \times 5$ Haralick features are extracted from this matrix. Therefore, total number $\left(\mathrm{N}_{\mathrm{f}}\right)$ of candidate color and texture features are reduced significantly:

$$
\text { Angular second momemnt }=\sum_{i} \sum_{j} p(i, j)^{2}
$$

$$
\begin{gathered}
\text { Constrast }=\sum_{\mathrm{n}=0}^{\mathrm{N}_{\mathrm{g}}-1} \mathrm{n}^{2}\left\{\sum_{\mathrm{i}=1}^{N_{\mathrm{g}}} \sum_{\mathrm{j}=0}^{N_{\mathrm{g}}} \mathrm{p}(\mathrm{i}, \mathrm{j})\right\},|\mathrm{i}=\mathrm{j}|=\mathrm{n} \\
\text { Correlation }=\frac{\sum_{\mathrm{i}} \sum_{\mathrm{j}}(\mathrm{i}, \mathrm{j}) \mathrm{p}(\mathrm{i}, \mathrm{j})-\mu_{\mathrm{z}} \mu_{\mathrm{y}}}{\sigma_{\mathrm{z}} \sigma_{\mathrm{y}}}
\end{gathered}
$$

Inverse differece moment $=\sum_{i} \sum_{j} \frac{p(i, j)}{1+(i-j)^{2}}$

$$
\text { Variance }=\sum_{i} \sum_{j}(p(i, j)-\mu)^{2} p(i, j)
$$

$\alpha$-cut implemented IT2FCM (IT2FCM $\alpha$ ) for segmentation: Once the feature extraction step is completed, a fast and robust algorithm to perform texture segmentation/extraction is needed. To reach this objective, researchers have proposed an adaptive region based color texture segmentation algorithm based on $\alpha$-cut implemented IT2FCM clustering. Although, fuzzy clustering algorithm with cluster cores has some good clustering properties in image it cannot define the cluster cores for two overlapping areas. Therefore to define more general cluster cores for any overlapping areas in image, researchers propose $\alpha$-cut implemented IT2FCM clustering algorithm denoted by ITFCM as follows. The main contribution of this algorithm is to utilize $\alpha$-plane representation concept in type- 2 fuzzy for calculating centriod type reduction in IT $2 \mathrm{FCM} \alpha$ and extended method presented in (Hwang and Rhee, 2007). This cluster core concept can be extended to more general way such that if the membership value $\mu_{\mathrm{ij}}$ of the data point $\mathrm{x}_{\mathrm{j}}$ in the ith cluster is larger than a given value then the point $x_{j}$ will exactly belong to the ith cluster with the membership value of 1 and ${\underline{\mu^{\prime} j}}=0$ and $\bar{\mu}_{i^{\prime} j}=0$ for all $i \neq i$. A simple rule to guarantee that no two of these c cluster cores will overlap is $0.5 \leq \alpha \leq 1$. The cluster cores generated by IT 2 FCM $\alpha$ can be determined by a relative distance measure with:

$$
\begin{aligned}
& \overline{\mathrm{u}}_{\mathrm{j}}\left(\mathrm{x}_{\mathrm{i}}\right)= \begin{cases}\frac{1}{\sum_{\mathrm{k}=1}^{\mathrm{c}}\left(\mathrm{d}_{\mathrm{ji}} / \mathrm{d}_{\mathrm{ki}}\right)^{2 / \mathrm{m}\left(\mathrm{m}_{1}-1\right)},}, & \text { if } \frac{1}{\sum_{\mathrm{k}=1}^{\mathrm{c}}\left(\mathrm{d}_{\mathrm{ji}} / \mathrm{d}_{\mathrm{ki}}\right)}<\frac{1}{\mathrm{c}} \\
\frac{1}{\sum_{\mathrm{k}=1}^{\mathrm{c}}\left(\mathrm{d}_{\mathrm{ji}} / \mathrm{d}_{\mathrm{ki}}\right)^{2 / \mathrm{m}\left(\mathrm{m}_{2}-1\right)}}, & \text { otherwise }\end{cases} \\
& \underline{\mathrm{u}}_{\mathrm{j}}\left(\mathrm{x}_{\mathrm{i}}\right)= \begin{cases}\frac{1}{\sum_{\mathrm{k}=1}^{\mathrm{c}}\left(\mathrm{d}_{\mathrm{ji}} / \mathrm{d}_{\mathrm{ki}}\right)^{2 / \mathrm{m}\left(\mathrm{m}_{1}-1\right)},} & \text { if } \frac{1}{\sum_{\mathrm{k}=1}^{\mathrm{c}}\left(\mathrm{d}_{\mathrm{ji}} / \mathrm{d}_{\mathrm{ki}}\right)} \geq \frac{1}{\mathrm{c}} \\
\frac{1}{\sum_{\mathrm{k}=1}^{\mathrm{c}}\left(\mathrm{d}_{\mathrm{ji}} / \mathrm{d}_{\mathrm{ki}}\right)^{2 / \mathrm{m}\left(\mathrm{m}_{2}-1\right)},} & \text { otherwise }\end{cases}
\end{aligned}
$$

Thus, the cluster cores generated by the proposed IT $2 \mathrm{FCM} \alpha$ algorithm are such that if the distance between the data point $\mathrm{x}_{\mathrm{j}}$ and ith cluster center $\mathrm{c}_{\mathrm{i}}$ is smaller than proportion of the vocal mean of the distances between $\mathrm{xj}$ and all cluster centers. Then, the data point $x_{j}$ will belong to the ith cluster with membership value of 1 . This will ensure that the cluster cores in IT2FCM $\alpha$ have different overlapping areas. 
Step 1: Compute $\underline{\mu}_{i j}^{(1)}$ and $\bar{\mu}_{i j}^{(1)}$.

Step 2: Update cluster centers with Eq. 22:

$$
\begin{aligned}
& \mathrm{v}_{\mathrm{j}}^{(1)}(\alpha)=\operatorname{Centroid}(\tilde{\mathrm{A}}(\alpha) \\
& =\sum_{\mathrm{u}_{1} \in \Theta J \mathrm{~J}_{1}} \ldots \sum_{\mathrm{u}_{\mathrm{N}} \in \omega \mathrm{dJ} \mathrm{X}_{\mathrm{N}}} \alpha / \frac{\sum_{\mathrm{i}=1}^{\mathrm{N}} \mathrm{x}_{\mathrm{i}} \mathrm{u}_{\mathrm{i}}}{\sum_{\mathrm{i}=1}^{\mathrm{N}} \mathrm{u}_{\mathrm{i}}} \\
& =\alpha /\left[{ }^{\alpha} v_{L},{ }^{\alpha} v_{R}\right] \\
& v_{j}^{(1)}(\alpha)=\frac{v_{L}^{(1)}+v_{R}^{(1)}}{2}
\end{aligned}
$$

This center-updating contains type-reduction and defuzzification.

Step 3: Perform type reduction and defuzzification, to compute ${ }^{\alpha} \mathrm{V}_{\mathrm{L}}$ and ${ }^{\alpha} \mathrm{V}_{\mathrm{R}}$ :

$$
\begin{gathered}
u_{j}^{(1)}\left(x_{i}\right)=\frac{u_{j}^{R}\left(x_{i}\right)+u_{j}^{L}\left(x_{i}\right)}{2}, j=1, \ldots, C \\
u_{j}^{R}\left(x_{i}\right)=\frac{\sum_{l=1}^{M} u j l\left(x_{i}\right)}{M}
\end{gathered}
$$

Where:

$$
u_{j 1}\left(x_{i}\right)= \begin{cases}\bar{u}_{j}\left(X_{i}\right), & \text { if } x_{i 1} u s e s \bar{u}_{j}\left(x_{i}\right) \text { for } v_{j}^{R} \\ \underline{u}_{j}\left(X_{i}\right), & \text { otherwise }\end{cases}
$$

And:

$$
\mathrm{u}_{\mathrm{j}}^{\mathrm{L}}\left(\mathrm{x}_{\mathrm{i}}\right)=\frac{\sum_{1=1}^{\mathrm{M}} \mathrm{u}_{\mathrm{jl}}\left(\mathrm{x}_{\mathrm{i}}\right)}{\mathrm{M}}
$$

Where:

$$
u_{j 1}\left(x_{i}\right)= \begin{cases}\bar{u}_{j}\left(X_{i}\right), & \text { if } x_{i 1} \text { uses } \bar{u}_{j}\left(x_{i}\right) \text { for } v_{j}^{L} \\ \underline{u}_{j}\left(X_{i}\right), & \text { otherwise }\end{cases}
$$

Step 4: If $\left(u_{j}\left(x_{i}\right)>u_{k}\left(x_{i}\right)\right)$, for $k=1, \ldots, C$ and $j \neq k$ then $x_{i}$ is assigned to cluster $j$.

Step 5: If $\max _{i}\left\|{ }^{\alpha} \mathrm{V}_{i}^{(1+1)}-{ }^{\alpha} \mathrm{V}_{i}^{(1)}\right\|<\varepsilon$ then stop; else $1=1+1$ and goto Step 2.

\section{RESULTS AND DISCUSSION}

A large number of experiments were carried out to analysis the performance of the proposed color texture segmentation algorithm using MATLAB. To demonstrate the capability of the proposed method, only three images (namely Rimg_1.jpg, Rimg_2.jpg, Rimg_3.jpg) are evaluated in detail to highlight the advantage of the proposed approach. The proposed algorithm influences the parameter, fuzziness index $\mathrm{m}$ and the parameter $\alpha$. Each set of experiment is carried out with different test parameters (GL, $\mathrm{HL}$ and $\left.\mathrm{r}_{1}, \mathrm{r}_{2}\right)$ in ICICM and $\left(\mathrm{m}_{1}, \mathrm{~m}_{2}\right.$ ) in IT2FCM $\alpha$. During every test, the features contrast, correlation, inverse difference moment, variance and angular second moment are extracted in ICICM of the image. Three set of experiments are carried out. The first set of experiment are attempted to evaluate the proposed approach to segment the natural color-texture images because natural images are having more number of overlapping areas. The second set of experiment are attempted to evaluate efficiency of the algorithm in composite texture. Each set of experiment is carried out with different $5 \times 1=5$ times on the image withtest parameters using ICICM. Such texture segmentation is carried out different settings as mentioned above. If $\alpha$ parameter value is 1 , the results will be exactly the same as the IT2FCM when $\alpha$ decreases from 1 , the volume of the cluster core increases. A psycho-visual evaluation of segmentation algorithm using human observars was proposed in (Shaffrey et al., 2002) the test was designed to visually compare segmentations results.

Figure 2 shows the overall benchmark performance of proposed methodology. The proposed method is a hybrid approach of ICICM and IT2FCM $\alpha$ algorithm. So, researchers have to set the parameter values for ICICM algorithm (GL, $\mathrm{HL}$ and $\mathrm{r}_{1}, \mathrm{r}_{2}$ ) and IT2FCM $\alpha$ algorithm $\left(\mathrm{m}_{1}, \mathrm{~m}_{2}, \alpha\right)$ (Vadivel et al., 2007). The researchers have proposed that the best combination of (HL and GL) is $(2,6)$ and $(4,4)$ while $\left(\mathrm{r}_{1}, \mathrm{r}_{2}\right)$ combinations in the range $(0.05,0.8)$ to $(0.15,0.9)$. In the experiment researchers also fixed the same combination with different values of $\alpha$ and $\mathrm{m}_{1}, \mathrm{~m}_{2}$ because these two are the influence factors in IT $2 \mathrm{FCM} \alpha$ algorithm. When researchers apply any algorithm in texture segmentation researchers have to consider two texture properties: texture boundary and homogeneity property of texture. Researchers experimented with the parameter value $\alpha=0.9$ and three combination of $\mathrm{m}_{1}, \mathrm{~m}_{2}$ such as $(2,4),(6,8)$ and $(8,10)$ and it over segments the images and also boundaries are lost. When parameter $\alpha=0.7$ and for three combination of $\mathrm{m}_{1}, \mathrm{~m}_{2}$ as $(2,4),(6,8)$ and $(8,10)$, number of segments are greatly reduced but the pixels of different textures are grouped together. This improper grouping among different texture regions lost the homogeneity property of textures. Then, researchers experimented for $\alpha=0.5$ and combination of $\mathrm{m}_{1}, \mathrm{~m}_{2}$ as $(2,4),(6,8)$ and $(8,10)$, respectively. But in $(6,8)$ and $(8,10)$ for both specification the color texture have segmented with very good pixel-level clarity. The obtained color texture regions are shown in column 2 nd and 5th column of Fig. 2. 
Res. J. Applied Sci., 7 (5): 258-265, 2012

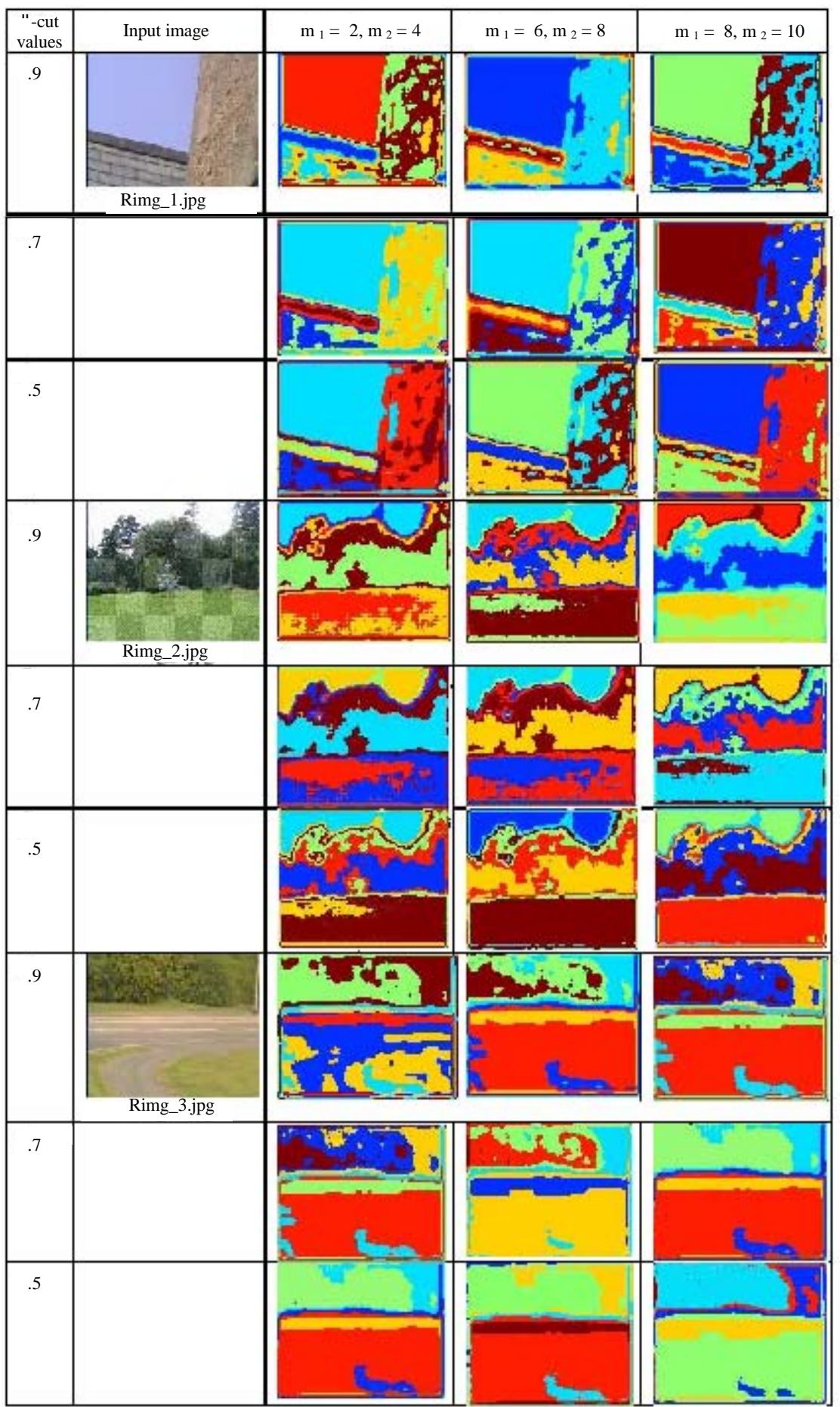

Fig. 2: Segmentation result for different combination of $\alpha \mathrm{m}_{1}, \mathrm{~m}_{2}$ with $\mathrm{r}_{1}=0.05, \mathrm{r}_{2}=0.08$ and $\mathrm{HL}=2 \mathrm{GL}=6$

The third set of experiment is intended to provide a comparative analysis of the proposed approach with the exiting methods such as FCM $\alpha$, IT2FCM to determine the effectiveness of the proposed approach. Figure 3 shows the segmentation result obtained in the experiment.
Figure 3a shows the original images with color textured regions. Segmented images with $\operatorname{FCM} \alpha$, IT2FCM, IT2FCM $\alpha$ are shown in Fig. 3b-d, respectively and their segment count is also mentioned in row 3 and 5 . 


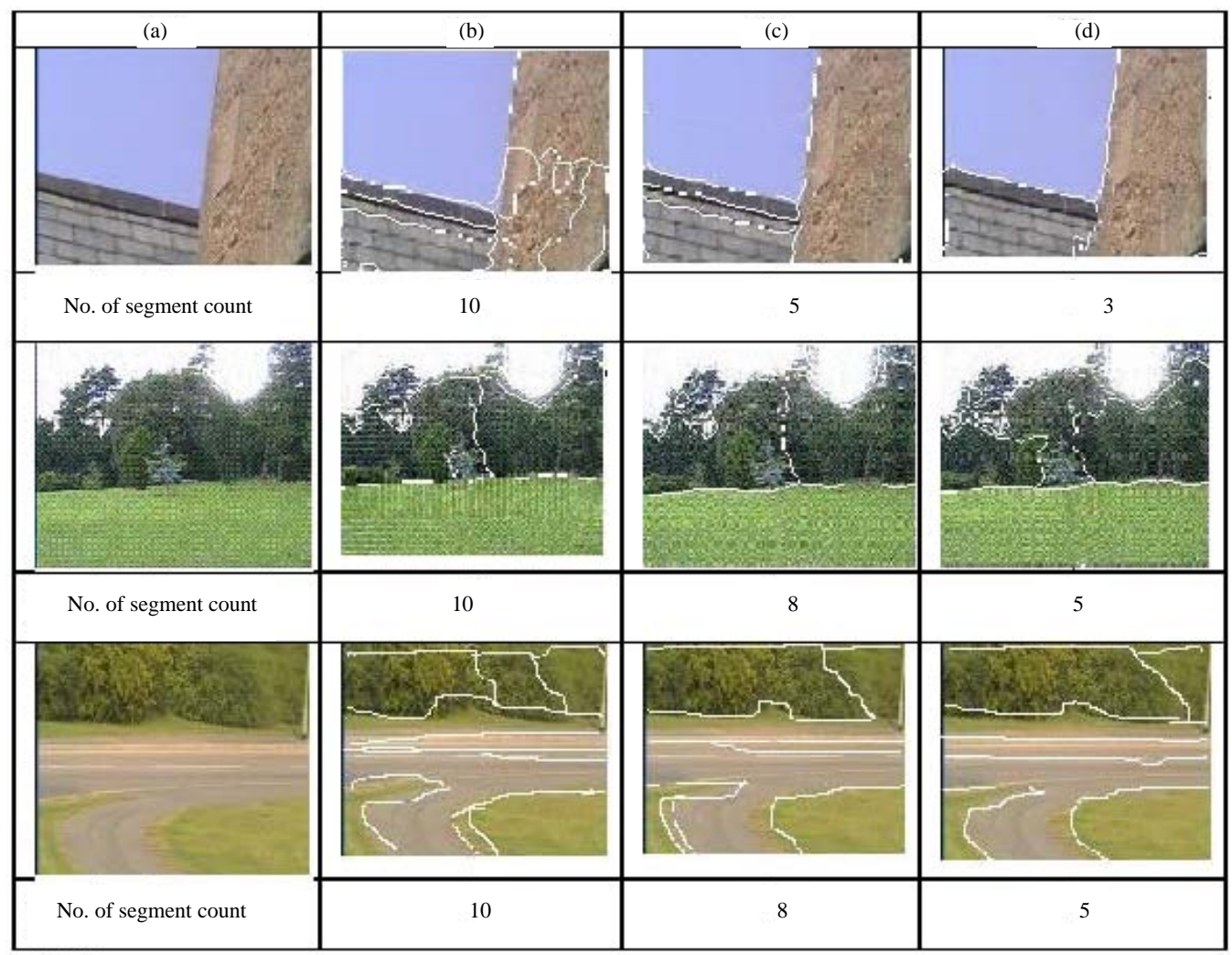

Fig. 3: Segmentation results of; a) original images based on; b) FCM $\alpha$; c) IT2FCM and d) IT2FCM $\alpha$. Row 3, 5, 7 shows the number of segment count

\section{CONCLUSION}

In this study, researchers have proposed $\alpha$-cut implemented interval type-2 fuzzy clustering algorithm for color-texture image segmentation. The concept of $\alpha$-cut implementation can be used to form cluster cores such as the data points inside the cluster core will have the membership value of 1. Moreover, IT2FCM $\alpha$ is suitable for segment overlapping areas in the natural and composite texture images with less computational complexity as FCM.

The proposed method has been compared with existing methods such as FCM, FCM $\alpha$ and IT2FCM. It is found that the computation complexity has been reduced in this method because only one integrated color intensity color matrix is used. From the experiments it is found that if the $\alpha$-cut value with 0.5 and 0.6 with $\mathrm{m}_{1}, \mathrm{~m}_{2}$ value $(6,8)$ and $(8,10)$ give good texture segmentation result. Furthermore, the approach could obtain better segmentation results with less number of regions as well as providing more homogeneous segmented regions. Applying and analyzing the performance of IT $2 \mathrm{FCM} \alpha$ in content based image retrieval system and segmentation of medical images can be focused for future research.

\section{ACKNOWLEDGEMENT}

The researchers would like to acknowledge the Tijuana Institute of Technology and Baja California Autonomous University, Tijuana Campus, Mexico for providing the Interval Type-2 Fuzzy toolbox.

\section{REFERENCES}

Chuang, K.S., H.L. Tzeng, S. Chen, J. Wu and T.J. Chen, 2006. Fuzzy c-means clustering with spatial information for image segmentation. Comput. Med. Imaging Graph., 30: 9-15.

Du, R. and H.J. Lee, 2009. A modified-FCM segmentation algorithm for brain MR images. Proceedings of the International Conference on Hybrid Information Technology, August 27-29, 2009, Daejeon, Korea. 
Fan, J.L., W.Z. Zhen and W.X. Xie, 2003. Suppressed fuzzy c-means clustering algorithm. Pattern Recog. Lett., 24: 1607-1612.

Hamrawi, H., S. Coupland and R. John, 2010. A novel alpha-cut representation for type- 2 fuzzy sets. Proceedings of the International conference on Fuzzy Systems, July 18-23, 2010, Barcelona, Spain, pp: $18-20$.

Haralick, R.M., K. Shanmugam and I.H. Dinstein, 1973. Textural features for image classification. IEEE Trans. Syst. Man Cybernet, 3: 610-621.

Hwang, C. and F.C.H. Rhee, 2007. Uncertain fuzzy clustering: Interval type-2 fuzzy approach to CMeans. Trans. Fuzzy Syst., 15: 1-6.

Ilea, D.E. and P.F. Whelan, 2011. Image segmentation based on the Integration of colour-texture descriptors: A review. Pattern Recognit., 44: 2479-2501.

Innocent, P. and R. John, 2002. Type 2 fuzzy diagnosis. Int. Conf. Fuzzy Syst., 2: 1326-1330.

Karnik, N., J. Mendel and Q. Liang, 2002. Type-II fuzzy logic systems. Trans. Fuzzy Syst., 10: 643-658.

Liang, Q. and J. Mendel, 2001. MPEG VBR video traffic modeling and classification using fuzzy techniques. Trans. Fuzzy Syst., 9: 183-193.

Liu, F., 2008. An efficient centroid type-reduction strategy for general type-2 fuzzy logic system. Inf. Sci., 178: 2224-2236.

Maheswari, G.U., K. Ramar, D. Manimegalai and V. Gomathi, 2011. An adaptive region based color texture segmentation using fuzzified distance metric. Applied Soft Comput., 11: 2916-2924.

Maity, S. and J. Sil, 2009. Color image segmentation using type-2 fuzzy sets. Int. J. Comp. Electr. Eng., 1: 1793-8163.

Pal, N.R., K. Pal, J.M. Keller and J.C. Bezdek, 2005. A possibilistic fuzzy c-means clustering algorithm. IEEE Trans. Fuzzy Syst., 13: 517-530.
Shaffrey, C.W., I.H. Jermyn and N.G. Kingsbury, 2002. Psychovisual evaluation of image segmentation algorithms. Proceedings of the Advanced Concepts for Intelligent Vision Systems, September, 2002, Ghent, Belgium, pp: S001-S007.

Shokouhifar, M. and F. Farokhi, 2010. Feature selection using supervised fuzzy C-means algorithm with ant colony. Proceedings of the 3rd International Conference on Machine Vision, December 28-30, 2010, Hong Kong, China.

Tlig, L., M. Sayadi and F. Fnaeich, 2010. A new descriptor for textured image segmentation based on fuzzy type- 2 clustering approach. Proceedings of the 2 nd International Conference on Image Processing Theory Tools and Applications, July 7-10, 2010, Paris, France, pp: 258-263.

Vadivel, A., S. Sural and A.K. Majumdar, 2007. An integrated color and intensity co-occurrence matrix. Pattern Recog. Lett., 28: 974-983.

Wang, S.T., F.L. Chung, Y.Y. Li, D.W. Hu and X.S. Wu, 2005. A new gaussian noise filter based on interval type 2 fuzzy systems. Soft Comput. Fusion Foundat. Methodol. Appl., 9: 398-406.

Yang, M.S. and K.L.Wu, 2003. A similarity based robust clustering method. Trans. Pattern Anal. Mach. Intell., 26: 43-448.

Yang, M.S., K.L. Wu, J.N. Hsieh and J. Yu, 2008. Alpha-cut implemented fuzzy clustering algorithms and switching. Cybern. Syst. Man Part B: Cyber. Trans., 38: 588-603.

Zadeh, L.A., 1975. The concept of a linguistic variable and its application to approximate reasoning-I. Inform. Sci., 8: 199-249.

Zhu, L., F.L. Chung and S. Wang, 2009. Generalized Fuzzy C-Means Clustering Algorithm With IEEE Trans. Syst. Man Cybern. B Cybern., 39: 578-591. 